\title{
An Application of Semigroup Theory to a Fragmentation Equation
}

\author{
P.N. Blair, W. Lamb and I.W. Stewart
}

\section{Introduction}

The process of fragmentation arises in many physical situations, including polymer degradation, droplet breakage and rock crushing and grinding. Under suitable assumptions, the evolution of the size distribution $c(x, t)$, where $x$ represents particle size and $t$ is time, may be described by the linear integro-differential equation

$$
\frac{\partial c(x, t)}{\partial t}=-a(x) c(x, t)+\int_{x}^{\infty} a(y) b(x, y) c(y, t) d y, x>0, t>0
$$

see [1] for details. In (1), $a(x)$ describes the rate at which a particle of size $x$ fragments and $b(x, y)$ represents the rate of production of particles of size $x$ due to the break-up of particles of size $y>x$. For the total mass in the system to remain constant during fragmentation, $b$ must satisfy the condition

$$
\int_{0}^{y} x b(x, y) d x=y
$$

Here we consider the case

$$
a(x)=x^{\alpha+1}, b(x, y)=(\nu+2)(x / y)^{\nu} y^{-1},
$$

where $\alpha \in \mathbb{R}$ and $-2<\nu \leq 0$. The restriction $\nu>-2$ is mathematical to ensure integrability whereas $\nu \leq 0$ is a physical property of the system that is explained in [1]. With these rate functions, equation (1) becomes

$$
\frac{\partial c(x, t)}{\partial t}=-x^{\alpha+1} c(x, t)+(\nu+2) \int_{x}^{\infty}(x / y)^{\nu} y^{\alpha} c(y, t) d y, x>0, t>0 .
$$

Our aim is to show that the questions of existence, uniqueness and mass conservation of solutions to (3) can be resolved by using the theory of

The work of the first author is supported by a Scholarship from the Carnegie Trust for the Universities of Scotland. 
semigroups of linear operators. For certain values of the parameters, the equation can be dealt with immediately by simply quoting previously established results. To cater for the remaining values, we use the idea of similar semigroups [2, p.43] to determine the required information on solutions. In the case when $\alpha>-1$, we also obtain an explicit formula for the semigroup associated with the equation. This yields a closed-form expression for the solution that coincides with that given in [1].

\section{The Abstract Problem}

Before semigroup theory can be applied, the initial-value problem associated with equation (3) is reformulated as an abstract Cauchy problem (ACP) of the form

$$
\frac{d}{d t} c(t)=A_{\nu, \alpha}[c(t)], t>0, c(0)=c_{0},
$$

where the operator $A_{\nu, \alpha}$ is defined on suitable functions $f$ by

$$
\left(A_{\nu, \alpha} f\right)(x)=-x^{\alpha+1} f(x)+(\nu+2) \int_{x}^{\infty}(x / y)^{\nu} y^{\alpha} f(y) d y, x>0
$$

As solutions to (3) are expected to be mass-conserving, an appropriate Banach space in which to study (4) is

$$
Y=\left\{f:\|f\|=\int_{0}^{\infty} x|f(x)| d x<\infty\right\}
$$

In this case, a natural domain for $A_{\nu, \alpha}$ is

$$
D\left(A_{\nu, \alpha}\right)=\left\{f \in Y: x^{\alpha+1} f \in Y\right\}
$$

since a routine calculation shows that $A_{\nu, \alpha} f \in Y$ whenever $f \in D\left(A_{\nu, \alpha}\right)$.

We note that the solution of (4) is interpreted as a strongly continuously differentiable $Y$-valued function of $t$. Such a solution is sought in the form $c(t)=S_{\nu, \alpha}(t) c_{0}, t \geq 0$, where $\left\{S_{\nu, \alpha}(t)\right\}_{t \geq 0}$ is a strongly continuous semigroup of linear operators on $Y$ that is generated by some extension of $\left(A_{\nu, \alpha}, D\left(A_{\nu, \alpha}\right)\right)$. This semigroup is said to be substochastic if $S_{\nu, \alpha}(t) \geq 0$ and $\left\|S_{\nu, \alpha}(t)\right\| \leq 1$ for each $t \geq 0$. If, additionally, $\left\|S_{\nu, \alpha}(t) f\right\|=\|f\|$ for all $t \geq 0$ and $f \in Y_{+}$, where $Y_{+}$is the cone of non-negative a.e. functions in $Y$, then $\left\{S_{\nu, \alpha}(t)\right\}_{t \geq 0}$ is called a stochastic semigroup. 


\section{Existing Results}

For the case $\alpha=-1$, the operator $A_{\nu,-1}$ is bounded on $Y$ and hence is the infinitesimal generator of a uniformly continuous semigroup $\left\{S_{\nu,-1}(t)\right\}_{t \geq 0}$ on $Y$, where

$$
S_{\nu,-1}(t)=\sum_{k=0}^{\infty} A_{\nu,-1}^{k} t^{k} / k !, t \geq 0 .
$$

As shown in [3, Lemma 2.4], this semigroup is stochastic and this, together with [2, p. 145, Proposition 6.2], establishes that the corresponding ACP has a unique, non-negative, mass-conserving solution $c(t)=S_{\nu,-1}(t) c_{0}$ for all $t \geq 0$ and $c_{0} \in Y_{+}$.

When $\alpha>-1$, the functions $a$ and $b$ defined by (2) satisfy the constraints imposed in [4]. Consequently, in this case, the semigroup $\left\{S_{\nu, \alpha}(t)\right\}_{t>0}$ can be obtained by means of a truncation/limit procedure which takes the form

$$
\begin{aligned}
& S_{\nu, \alpha}^{(n)}(t)=\sum_{k=0}^{\infty}\left(A_{\nu, \alpha} P_{n}\right)^{k} t^{k} / k !=I-P_{n}+\exp \left(A_{\nu, \alpha} t\right) P_{n}, t \geq 0, \\
& S_{\nu, \alpha}(t) f=\lim _{n \rightarrow \infty} S_{\nu, \alpha}^{(n)}(t) f, f \in Y, t \geq 0,
\end{aligned}
$$

where

$$
\left(P_{n} f\right)(x)= \begin{cases}f(x), & \text { if } 0<x<n \\ 0, & \text { if } x \geq n .\end{cases}
$$

Theorem 1. The semigroup $\left\{S_{\nu, \alpha}(t)\right\}_{t \geq 0}$ defined via (5)-(7) is stochastic and is generated by an extension $\left(\tilde{A}_{\nu, \alpha}, D\left(\tilde{A}_{\nu, \alpha}\right)\right)$ of $\left(A_{\nu, \alpha}, D\left(A_{\nu, \alpha}\right)\right)$. Hence the $A C P$

$$
\frac{d}{d t} c(t)=\tilde{A}_{\nu, \alpha}[c(t)], t>0, c(0)=c_{0}
$$

has a unique, non-negative, mass-conserving solution for each non-negative $c_{0} \in D\left(\tilde{A}_{\nu, \alpha}\right)$.

Proof. See [4, Sections 2-5]

One drawback to the approach used in [4] is that it cannot be applied when $\alpha<-1$. However, in [5] an alternative strategy involving the KatoVoigt perturbation theorem is used to analyse (4) for any $\alpha \neq-1$ but under the additional constraint that $\nu=0$.

Theorem 2. For each $\alpha \neq-1$ there exists a smallest substochastic semigroup $\left\{S_{0, \alpha}(t)\right\}_{t \geq 0}$ on $Y$ generated by an extension of $\left(A_{0, \alpha}, D\left(A_{0, \alpha}\right)\right)$. Furthermore, if $\alpha>-1$, then this semigroup is stochastic with generator $\left(\bar{A}_{0, \alpha}, D\left(\bar{A}_{0, \alpha}\right)\right)$ (i.e. the closure of $\left(A_{0, \alpha}, D\left(A_{0, \alpha}\right)\right)$ ), whereas if $\alpha<-1$, the generator is a proper extension of $\left(\bar{A}_{0, \alpha}, D\left(\bar{A}_{0, \alpha}\right)\right)$. In the latter case, for each $f \in Y_{+}$, there exists $t>0$ such that $\left\|S_{0, \alpha}(t) f\right\|<\|f\|$, i.e. a mass loss occurs.

Proof. See [5, Sections 4,5]. 


\section{Extension of Results}

We rely on the idea of similar semigroups to extend the results stated in Theorem 2 to the case when $\nu \neq 0$. Motivated by transformations used in [1], we introduce an operator, $R_{\nu}$, defined on $Y$ by

$$
\left(R_{\nu} f\right)(x)=x^{-2 \nu /(\nu+2)} f\left(x^{2 /(\nu+2)}\right) .
$$

It is a straightforward matter to show that $R_{\nu}$ is a homeomorphism from $Y$ onto $Y$. Moreover,

$$
R_{\nu} A_{\nu, \alpha} R_{\nu}^{-1}=A_{0, \beta}, \quad \text { where } \beta=(2 \alpha-\nu) /(\nu+2) .
$$

Consequently, as pointed out in [1], it is possible to transform the original multiple fragmentation problem into an equivalent binary problem.

Theorem 3. For each $\alpha \neq-1$ and $\nu \in(-2,0]$ there exists a smallest substochastic semigroup $\left\{S_{\nu, \alpha}(t)\right\}_{t \geq 0}$ on $Y$ generated by an extension of $\left(A_{\nu, \alpha}, D\left(A_{\nu, \alpha}\right)\right)$. Furthermore, if $\alpha>-1$, then this semigroup is stochastic with generator $\left(\bar{A}_{\nu, \alpha}, D\left(\bar{A}_{\nu, \alpha}\right)\right)$, whereas if $\alpha<-1$, the generator is a proper extension of $\left(\bar{A}_{\nu, \alpha}, D\left(\bar{A}_{\nu, \alpha}\right)\right)$. In the latter case, for each $f \in Y_{+}$, there exists $t>0$ such that $\left\|S_{\nu, \alpha}(t) f\right\|<\|f\|$, i.e. a mass loss occurs.

Proof. Since $S_{\nu, \alpha}(t)=R_{\nu}^{-1} S_{0, \beta}(t) R_{\nu} \forall t \geq 0$, where $\beta=(2 \alpha-\nu) /(\nu+2)$, and $\beta>-1 \Leftrightarrow \alpha>-1$, the result follows from Theorem 2 .

Corollary 1. If $\alpha>-1$ and $\nu \in(-2,0]$ then the semigroups in Theorems 1 and 3 are identical.

Proof. This follows since the closure of the operator $\left(A_{\nu, \alpha}, D\left(A_{\nu, \alpha}\right)\right)$ (which exists from Theorem 3 ) can be shown to be the infinitesimal generator of the semigroup defined by (5)-(7).

\section{Exact Solutions}

Following an approach similar to that used in [3] for the case $\alpha=-1$, we now obtain an explicit formula for the semigroup $\left\{S_{\nu, \alpha}(t)\right\}_{t \geq 0}$ when $\alpha>-1$. Again, it is convenient to apply a similarity transformation to simplify $A_{\nu, \alpha}$. Defining the operator $W_{\nu, \alpha}$ and Banach space $Y_{\nu, \alpha}$ by

$$
\begin{aligned}
& \left(W_{\nu, \alpha} f\right)(x)=x^{-\nu /(\alpha+1)} f\left(x^{1 /(\alpha+1)}\right), \\
& Y_{\nu, \alpha}=\left\{f:\|f\|_{\nu, \alpha}=\int_{0}^{\infty} x^{(\nu-\alpha+1) /(\alpha+1)}|f(x)| d x<\infty\right\}
\end{aligned}
$$

it can be shown that $W_{\nu, \alpha}$ is a homeomorphism from $Y$ onto $Y_{\nu, \alpha}$. Moreover, $W_{\nu, \alpha} A_{\nu, \alpha} W_{\nu, \alpha}^{-1}=A_{\gamma}$ where

$$
\left(A_{\gamma} f\right)(x)=-x f(x)+\frac{2}{\gamma} \int_{x}^{\infty} f(y) d y
$$


with $\gamma=2(\alpha+1) /(\nu+2)$ and $D\left(A_{\gamma}\right)=\left\{f \in Y_{\nu, \alpha}: x f \in Y_{\nu, \alpha}\right\}$.

Lemma 1. If $\alpha>-1$ then the operator

$$
\left(\bar{A}_{\gamma}, D\left(\bar{A}_{\gamma}\right)\right)=\left(W_{\nu, \alpha} \bar{A}_{\nu, \alpha} W_{\nu, \alpha}^{-1}, W_{\nu, \alpha}\left[D\left(\bar{A}_{\nu, \alpha}\right]\right)\right.
$$

generates a stochastic semigroup $\left\{S_{\gamma}(t)\right\}_{t \geq 0}=\left\{W_{\nu, \alpha} S_{\nu, \alpha}(t) W_{\nu, \alpha}^{-1}\right\}_{t \geq 0}$ on $Y_{\nu, \alpha}$. Moreover,

$$
S_{\gamma}(t) f=\lim _{n \rightarrow \infty}\left(I-P_{n^{\alpha+1}}+\exp \left(A_{\gamma} t\right) P_{n^{\alpha+1}}\right) f, f \in Y_{\nu, \alpha},
$$

where $P_{n^{\alpha+1}}$ is defined by (7).

Proof. This follows from Theorem 3, Corollary 1 and formulae (5)-(7).

An explicit formula for $S_{\gamma}(t)$ can now be obtained using (8) and the power series definition of $\exp \left(A_{\gamma} t\right)$. First we require a formula for positive iterates of the operator $A_{\gamma}$.

Lemma 2. For each $k=1,2,3, \ldots$,

$$
\begin{aligned}
\left(A_{\gamma}^{k} f\right)(x) & =(-x)^{k} f(x)+ \\
& \frac{2(-1)^{k-1}}{\gamma} \sum_{j=1}^{k}\left(\begin{array}{c}
k \\
j
\end{array}\right)\left(1-\frac{2}{\gamma}\right)_{j-1} x^{k-j} \int_{x}^{\infty} \frac{(y-x)^{j-1}}{(j-1) !} f(y) d y,
\end{aligned}
$$

where $(c)_{k}=c(c+1) \ldots(c+k-1)$.

Proof. The proof follows by induction.

Lemma 3. For each $t>0$ and $f \in Y_{\nu, \alpha}$,

$$
\left(S_{\gamma}(t) f\right)(x)=e^{-x t}\left(f(x)+\frac{2 t}{\gamma} \int_{x}^{\infty}{ }_{1} F_{1}\left(1-\frac{2}{\gamma}, 2 ; t(x-y)\right) f(y) d y\right)
$$

where ${ }_{1} F_{1}$ denotes the confluent hypergeometric function.

Proof. This can be deduced from (8) and Lemma 2.

Theorem 4. For each $\alpha>-1, \nu \in(-2,0], t>0$ and $f \in Y$,

$$
\begin{aligned}
& \left(S_{\nu, \alpha}(t) f\right)(x)=e^{-t x^{\alpha+1}}[f(x)+ \\
& \left.\quad(\nu+2) t \int_{x}^{\infty}{ }_{1} F_{1}\left(\frac{\alpha-\nu-1}{\alpha+1}, 2 ; t\left(x^{\alpha+1}-y^{\alpha+1}\right)\right)\left(\frac{x}{y}\right)^{\nu} y^{\alpha} f(y) d y\right] .
\end{aligned}
$$

Moreover, the domain $D\left(A_{\nu, \alpha}\right)$ is positively invariant under the semigroup $\left\{S_{\nu, \alpha}(t)\right\}_{t \geq 0}$.

Proof. The formula given for $S_{\nu, \alpha}(t)$ follows from Lemma 3 and the fact that $S_{\nu, \alpha}(t)=W_{\nu, \alpha}^{-1} S_{\gamma}(t) W_{\nu, \alpha}$. The invariance property can be established by a direct calculation. 
The invariance of $D\left(A_{\nu, \alpha}\right)$ leads immediately to the following more satisfactory version of Theorem 2 .

Corollary 2. If $\alpha>-1$ and $\nu \in(-2,0]$ then the ACP (4) has the unique, non-negative mass-conserving solution $c(t)=S_{\nu, \alpha}(t) c_{0}$ for each non-negative $c_{0}$ in $D\left(A_{\nu, \alpha}\right)$.

\section{References}

1. E.D. McGrady and R.M. Ziff, "Shattering" transition in fragmentation, Phys. Rev. Lett. 58 (1987), 892-895.

2. K-J. Engel and R. Nagel, One-parameter semigroups for linear evolution equations, Springer-Verlag, New York, 1999.

3. W. Lamb and A.C. McBride, On a continuous coagulation and fragmentation equation with a singular fragmentation kernel, to appear in Recent contributions to evolution equations, Marcel Dekker Lecture Notes Ser.

4. D.J. McLaughlin, W. Lamb and A.C. McBride, A semigroup approach to fragmentation models, SIAM J. Math. Anal. 28 (1997), 1158-1172.

5. J. Banasiak, On an extension of the Kato-Voigt perturbation theorem for substochastic semigroups and its application, Taiwanese J.Math.5 (2001), 169-191. 